\title{
ON THE SUMMABILITY OF CERTAIN ORTHOGONAL DEVELOPMENTS OF NONLINEAR FUNCTIONALS
}

\author{
R. H. CAMERON AND C. HATFIELD, JR.
}

1. Introduction. Using Wiener's method of integration in function space, Cameron and Martin ${ }^{2}$ have constructed a set of closed orthonormal functionals in the space of nonlinear functionals which are Wiener measurable and whose squares are Wiener summable. It is the purpose of this paper to investigate the infinite-dimensional Abel summability of the orthogonal development of a nonlinear functional in terms of these orthogonal functionals.

The space of functions over which Wiener's integral operates is the space $C$ of functions $x(t)$ defined and continuous on $0 \leqq t \leqq 1$ and vanishing at $t=0$. The whole space has measure unity, and measure in this space is built up in exactly the same way as ordinary Lebesgue measure, except that the definition of an interval and its measure are different.

Wiener defines an interval $Q$, or as he calls it, a "quasi-interval" in his space by the inequalities

$Q$ :

$$
\alpha_{j}<x\left(t_{j}\right)<\beta_{j}, \quad j=1, \cdots, n,
$$

where $t_{1}, \cdots, t_{n}$ is any finite set of numbers such that $0<t_{1}<t_{2}<\cdots$ $<t_{n} \leqq 1$. He defines the measure of $Q$ as

$$
\begin{aligned}
m(Q)=\left[\pi^{n} t_{1}\left(t_{2}-t_{1}\right) \cdots\left(t_{n}-t_{n-1}\right)\right]^{-1 / 2} \int_{\alpha_{n}}^{\beta_{n}} \cdots \int_{\alpha_{1}}^{\beta_{1}} \\
\cdot \exp \left[-\frac{\xi_{1}^{2}}{t_{1}}-\frac{\left(\xi_{2}-\xi_{1}\right)^{2}}{t_{2}-t_{1}}-\cdots-\frac{\left(\xi_{n}-\xi_{n-1}\right)^{2}}{t_{n}-t_{n-1}}\right] d \xi_{1} \cdots d \xi_{n},
\end{aligned}
$$

and shows that this definition is self-consistent and leads to a satisfactory measure for general sets, in accordance with the usual definition of Lebesgue measure. After measure is defined, the usual Lebesgue procedure gives a satisfactory definition of integral with all the usual properties except invariance under translations and simple behavior under magnifications. Measurability and summabil-

\footnotetext{
Presented to the Society, February 28, 1948; received by the editors February 16, 1948.

${ }^{1}$ Generalized harmonic analysis, Acta Math. vol. 55 (1930) pp. 117-258, esp. pp. 214-224.

2 The orthogonal development of non-linear functionals in series of Fourier-Hermite functionals, Ann. of Math. vol. 48 (1947) pp. 385-392.
} 
ity of functionals are defined as usual. It is convenient to denote the Wiener integral of a summable functional $F(x)$ over a measurable set $S$ by

$$
\int_{S}^{w} F(x) d_{w} x
$$

In particular, it has been shown that if

$$
F(x)=f\left\{\int_{0}^{1} \alpha_{1}(t) d x(t), \cdots, \int_{0}^{1} \alpha_{n}(t) d x(t)\right\},
$$

where $\left\{\alpha_{j}(t)\right\}, j=1,2, \cdots, n$, are orthonormal and of bounded variation, then provided the second member exists,

$$
\begin{aligned}
& \int_{C}^{w} F(x) d_{w} x \\
& =\pi^{-n / 2} \int_{-\infty}^{\infty} \cdots \int_{-\infty}^{\infty} f\left(u_{1}, \cdots, u_{n}\right) \exp \left(\sum_{k=1}^{n} u_{k}^{2}\right) d u_{1} \cdots d u_{n} .
\end{aligned}
$$

Convenient Fubini theorems enable us to interchange order of two Wiener integrals or a Wiener integral and an $n$-dimensional Lebesgue integral in the ordinary way. Thus the integral has the usual satisfactory manipulative properties. We denote by $L_{2}(C)$ the space of measurable complex functionals $F(x)$ such that

$$
\int_{C}^{w}|F(x)|^{2} d_{w} x<\infty
$$

An orthonormal set of functionals $\left\{F_{n}\right\}$ is of course defined to be a set such that

$$
\int_{C}^{w} F_{j}(x) \overline{F_{k}(x)} d_{w} x=\delta_{j k}, \quad j, k=1,2, \cdots,
$$

where $\delta_{j k}$ is the Kronecker delta, and such a set is closed if for each functional $G \in L_{2}(C)$ and a given $\epsilon>0$, there exists a set of constants $C_{1}, C_{2}, \cdots, C_{n}$ such that

$$
\int_{C}^{w}\left|G(x)-\sum_{j=1}^{n} C_{j} F_{j}(x)\right|^{2} d_{w} x<\epsilon .
$$

A C.O.N. (closed orthonormal) set of functionals can be constructed as follows. Let $\alpha_{1}(t), \alpha_{2}(t), \cdots$ be a C.O.N. set of real functions on $0 \leqq t \leqq 1$ and each $\alpha_{j}(t)$ of bounded variation, and let $H_{n}(u)$ be the 
partially normalized Hermite polynomials

$$
H_{n}(u)=(-1)^{n} 2^{-n / 2}(n !)^{-1 / 2} e^{u^{2}} \frac{d^{n}}{d u^{n}}\left(e^{-u^{2}}\right), \quad n=0,1,2, \cdots .
$$

Let

$\Phi_{m, p}(x)=H_{m}\left[\int_{0}^{1} \alpha_{p}(t) d x(t)\right], \quad m=0,1, \cdots ; p=1,2, \cdots$,

and

$$
\Psi_{m_{1}, \cdots, m_{p}}(x) \equiv \Psi_{m_{1}, \cdots, m_{p}, 0, \cdots, 0}(x)=\Phi_{m_{1}, 1}(x) \cdots \Phi_{m_{p}, p}(x) .
$$

This is called the Fourier-Hermite set of functionals. As the above notation indicates, any number of zero subscripts may be added at the end of the already given list of subscripts without changing $\Psi$, because $H_{0}(u) \equiv \Phi_{0, p}(x) \equiv 1$. Thus the entire set of the $\Psi_{m_{1}, \cdots, m_{p}}$ without repetition consists of

$$
\begin{aligned}
& \Psi_{0} ; \\
& \left\{\Psi_{m_{1}}\right\}, \\
& \left\{\Psi_{m_{1}, m_{2}}\right\}, \\
& \cdot \cdot \cdot \cdot \cdot m_{1}=0,1,2, \cdots, m_{2}=1,2, \cdots ; \\
& \left\{\Psi_{m_{1}, \cdots, m_{p}}\right\}, m_{i}=0,1,2, \cdots \text { for } i=1,2, \cdots, p-1, m_{p}=1,2, \cdots ;
\end{aligned}
$$

Despite our complicated set of subscripts, this is still only a countable set of functionals. It has been shown ${ }^{2}$ that this is a C.O.N. set over $L_{2}(C)$, Thus, if $F(x) \in L_{2}(C)$, its Fourier-Hermite coefficients are defined to be

$$
A_{m_{1}}, \cdots, m_{N}=\int_{c}^{w} F(x) \Psi_{m_{1}, \cdots, m_{N}}(x) d_{w} x
$$

and we have

$$
\lim _{N \rightarrow \infty} \int_{c}^{w}\left|F(x)-\sum_{m_{1}, \cdots, m_{N}=0}^{N} A_{m_{1}, \cdots, m_{N}} \Psi_{m_{1}, \cdots, m_{N}}(x)\right|^{2} d_{w} x=0 .
$$

Thus the Fourier-Hermite development of $F$ converges in the sense of a limit in the mean for $L_{2}(C)$ to the original functional. However, this does not tell us that it converges in any sense at all for any given fixed function $x$, just as convergence in the mean for a Fourier series 
does not provide any information at all about the behavior of the series at a specific point. The convergence of the Fourier series at a fixed point requires more specific information about the generating function at that point, and it is reasonable to expect that much more would be required in the case of the functional. It is not at all clear what the analogue of the notion of bounded variation should be in order for us to carry through a pointwise convergence theorem in $L_{2}(C)$. However, if we are content to establish summability of the Fourier series of a function at the specified point, then we do not need bounded variation at the point, but only continuity; and continuity does have a satisfactory analogue for functionals. Since our orthogonal functionals are given in terms of Hermite functions, our summability will depend on a formula for sums of products of Hermite functions. Such a formula has been given by Wiener, ${ }^{3}$ for $|t|<1,-\infty<x<\infty$, $-\infty<y<\infty$, namely,

$$
\begin{aligned}
\sum_{n=0}^{\infty} t^{n} \psi_{n}(x) \psi_{n}(y) & \equiv K(x, y, t) \\
= & {\left[\pi\left(1-t^{2}\right)\right]^{-1 / 2} \exp \left[\frac{4 x y t-\left(1+t^{2}\right)\left(x^{2}+y^{2}\right)}{2\left(1-t^{2}\right)}\right] }
\end{aligned}
$$

where

$$
\psi_{n}(x)=\pi^{-1 / 4} H_{n}(x) e^{-x^{2} / 2}, \quad n=0,1, \cdots .
$$

Wiener showed that this series converges absolutely when $|t|<1$ and that for fixed $t$ such that $|t|<1$, the series converges in the mean in $x$ and $y$ to its value. It also converges in the mean as a function of $x$ for almost all fixed $y$, and vice versa.

The fact that (1.2) involves powers of $t$ led us to investigate infinite-dimensional Abel summability for the Fourier-Hermite series. We now state our main theorem.

Theorem. Let $C$ be the space of all continuous functions $x(t)$ on $0 \leqq t \leqq 1$ such that $x(0)=0$. Let $F(x)=F(x(\cdot))$ be a bounded Wiener measurable functional (linear or nonlinear) for $x \in C$, and let $F(x)$ be continuous in the Hilbert topology at $x_{0} \in C$, that is,

$$
\left|F(x)-F\left(x_{0}\right)\right|<\epsilon \text { when } \int_{0}^{1}\left[x(t)-x_{0}(t)\right]^{2} d t<\delta^{2}(\epsilon) .
$$

\section{Let}

${ }^{3}$ N. Wiener, The Fourier integral, pp. 55-67. 


$$
\begin{aligned}
\Psi_{m_{1}, \cdots, m_{N}}(x)= & H_{m_{1}}\left(\int_{0}^{1} 2^{1 / 2} \cos \frac{\pi t}{2} d x(t)\right) \\
& \cdot H_{m_{2}}\left(\int_{0}^{1} 2^{1 / 2} \cos \frac{3 \pi t}{2} d x(t)\right) \\
& \cdots H_{m_{N}}\left(\int_{0}^{1} 2^{1 / 2} \cos \frac{(2 N-1) \pi t}{2} d x(t)\right)
\end{aligned}
$$

and let

$$
A_{m_{1}, \cdots, m_{N}}=\int_{C}^{w} F(x) \Psi_{m_{1}, \cdots, m_{N}}(x) d_{w} x
$$

then

$$
F\left(x_{0}\right)=\lim _{N \rightarrow \infty, \lambda \rightarrow 1-0} \sum_{m_{1}, \cdots, m_{N}=0}^{\infty} A_{m_{1}, \cdots, m_{N}} \lambda^{m_{1}+\cdots+m_{N}} \Psi_{m_{1}}, \cdots, m_{N}\left(x_{0}\right) .
$$

(Remark. The reader will note that we have specialized the functional $\Psi$ by taking the C.O.N. set $\left\{\alpha_{j}(t)\right\}$ to be

$$
\alpha_{j}(t)=2^{1 / 2} \cos \frac{(2 j-1) \pi t}{2}, \quad j=1,2, \cdots .
$$

It was found necessary to take advantage of the special properties of the $\cos (j-1 / 2) \pi t$. In particular, these functions satisfy the boundary conditions $\alpha_{j}(1)=\alpha_{j}^{\prime}(0)=0$. It is hoped that later investigations will make it possible to use less specialized $\alpha_{j}(t)$.)

Using the fact that the Abel mean is regular for one-dimensional series we shall prove also the following corollary on pointwise convergence:

CoROLLARY. If we assume, in addition to the hypotheses of the theorem, that for each positive integer $N$

$$
\sum_{m_{1}, \cdots, m_{N}=0}^{\infty} A_{m_{1}, \cdots, m_{N}} \Psi_{m_{1}, \cdots, m_{N}}\left(x_{0}\right)
$$

converges absolutely, then

$$
F\left(x_{0}\right)=\lim _{N \rightarrow \infty} \sum_{m_{1}, \cdots, m_{N}=0}^{\infty} A_{m_{1}, \cdots, m_{N}} \Psi_{m_{1}, \cdots, m_{N}}\left(x_{0}\right) .
$$

2. An integral expression for the difference $D\left(x_{0}\right)$ between a functional and its Fourier-Hermite partial sum. In order to prove our main theorem stated above we proceed to sum the Fourier-Hermite 
series with appropriate powers of $\lambda$ as the weight factors. Putting $x=y$ and $t=\lambda^{2}$ in (1.2) we get

$$
K(x, x, t)=\sum_{n=0}^{\infty} \lambda^{2 n} \psi_{n}^{2}(x)=\sum_{n=0}^{\infty} \lambda^{2 n} \pi^{-1 / 2} e^{-x^{2}} H_{n}^{2}(x),
$$

and hence the series

$$
\sum_{n=0}^{\infty} \lambda^{2 n} H_{n}^{2}(x)=\pi^{1 / 2} e^{x^{2}} K(x, x, t)
$$

converges absolutely when $|\lambda|<1$. If we replace $x$ by $\int_{0}^{1} \alpha_{j}(t) d x_{0}(t)$ and understand by $\alpha_{j}(t)$ the specialized functions of (1.4), then when $x \in C$,

$$
\begin{aligned}
& \prod_{j=1}^{N}\left[\sum_{m_{j}=0}^{\infty} \lambda^{2 m_{j}} H_{m_{j}}^{2}\left(\int_{0}^{1} \alpha_{j}(t) d x_{0}(t)\right)\right] \sum_{m_{1}, \cdots, m_{N}=0}^{\infty} \lambda^{2\left(m_{1}+\cdots+m_{N}\right)} H_{m_{1}}^{2}\left(\int_{0}^{1} \alpha_{1}(t) d x_{0}(t)\right) \\
& \cdots H_{m_{N}}^{2}\left(\int_{0}^{1} \alpha_{N}(t) d x_{0}(t)\right) \\
&=\sum_{m_{1}, \cdots, m_{N}=0}^{\infty} \lambda^{2\left(m_{1}+\cdots+m_{N}\right)} \Psi_{m_{1}, \cdots, m_{N}}^{2}\left(x_{0}\right)
\end{aligned}
$$

converges absolutely for $|\lambda|<1$. Since the functionals $\left\{\Psi_{m_{1}, \cdots, m_{N}}(x)\right\}$ form a C.O.N. set in the space $L_{2}(C)$, we may use the Riesz-Fischer theorem for that space. Thus

$$
\underset{M \rightarrow \infty}{\operatorname{L.I.M.}} \sum_{m_{1}, \cdots, m_{N}=0}^{M} \lambda^{m_{1}+\cdots+m_{N}} \Psi_{m_{1}, \cdots, m_{N}}\left(x_{0}\right) \Psi_{m_{1}, \cdots, m_{N}}(x)
$$

exists, since (2.1) shows that the sum of the squares of the coefficients of $\Psi_{m_{1}}, \cdots, m_{N}(x)$ in (2.2) converges. Since $F(x)$ is bounded and Wiener measurable, $F(x) \subset L_{2}(C)$, and this with the existence of (2.2) justifies the interchange of order of integration and summation in:

$$
\begin{gathered}
\int_{c}^{w} F(x) \sum_{m_{1}, \cdots, m_{N}=0}^{\infty} \lambda^{m_{1}+\cdots+m_{N}} \Psi_{m_{1}}, \cdots, m_{N}(x) \Psi_{m_{1}}, \cdots, m_{N}\left(x_{0}\right) d_{w} x \\
(2.3)=\sum_{m_{1}, \cdots, m_{N}=0}^{\infty} \lambda^{m_{1}+\cdots+m_{N}} \int_{c}^{w} F(x) \Psi_{m_{1}}, \cdots, m_{N}(x) d_{w} x \Psi_{m_{1}}, \cdots, m_{N}\left(x_{0}\right) \\
=\sum_{m_{1}, \cdots, m_{N}=0}^{\infty} \lambda^{m_{1}+\cdots+m_{N}} A_{m_{1}, \cdots, m_{N}} \Psi_{m_{1}, \cdots, m_{N}}\left(x_{0}\right) .
\end{gathered}
$$


Thus the convergence of the last member of (2.3) for all $x_{0}$ and for $|\lambda|<1$ is established. In the same way that we proved the absolute convergence of the last member of (2.1) we can show that the first member (as well as the second and third) of the following expression converges absolutely:

$$
\begin{aligned}
\sum_{m_{1}}^{\infty}, \cdots, m_{N}=0 & \lambda^{m_{1}+\cdots+m_{N}} \Psi_{m_{1}}, \cdots, m_{N}(x) \Psi_{m_{1}}, \cdots, m_{N}\left(x_{0}\right) \\
= & \sum_{m_{1}, \cdots, m_{N}=0}^{\infty} \lambda^{m_{1}+\cdots+m_{N}} H_{m_{1}}\left(\int_{0}^{1} \alpha_{1}(t) d x(t)\right) \\
& \cdots H_{m_{N}}\left(\int_{0}^{1} \alpha_{N}(t) d x(t)\right) \\
& \cdot H_{m_{1}}\left(\int_{0}^{1} \alpha_{1}(t) d x_{0}(t)\right) \cdots H_{m_{N}}\left(\int_{0}^{1} \alpha_{N}(t) d x_{0}(t)\right) \\
= & \prod_{j=1}^{N}\left[\sum_{m_{j}=0}^{\infty} \lambda^{m_{j}} H_{m_{j}}\left(\int_{0}^{1} \alpha_{j}(t) d x(t)\right)\right. \\
\cdot & \left.H_{m_{j}}\left(\int_{0}^{1} \alpha_{j}(t) d x_{0}(t)\right)\right] .
\end{aligned}
$$

In view of (1.2) and (1.3) we have

$$
K(x, y, t)=\sum_{n=0}^{\infty} t^{n} \pi^{-1 / 2} e^{-\left(x^{2}+y^{2}\right) / 2} H_{n}(x) H_{n}(y),
$$

so that

$$
\begin{aligned}
\sum_{n=0}^{\infty} t^{n} H_{n}(x) H_{n}(y) & =\pi^{1 / 2} e^{\left(x^{2}+y^{2}\right) / 2} K(x, y, t) \\
& =\left(1-t^{2}\right)^{-1 / 2} \exp \left[\frac{2 x y t-t^{2}\left(x^{2}+y^{2}\right)}{1-t^{2}}\right] .
\end{aligned}
$$

Thus replacing $t, x, y$ by $\lambda, \int_{0}^{1} \alpha_{j}(t) d x_{0}(t), \int_{0}^{1} \alpha_{j}(t) d x(t)$ respectively in (2.5) and substituting in (2.4) we get

$$
\begin{aligned}
& \sum_{m_{1}, \cdots, m_{N}=0}^{\infty} \lambda^{m_{1}+\cdots+m_{N}} \Psi_{m_{1}}, \cdots, m_{N}(x) \Psi_{m_{1}, \cdots, m_{N}}\left(x_{0}\right) \\
&=\prod_{i=1}^{N}\left[\left(1-\lambda^{2}\right)^{-1 / 2} \exp \left\{\frac{2 \lambda u_{i} \xi_{i}-\lambda^{2}\left(u_{i}^{2}+\xi_{i}^{2}\right)}{1-\lambda^{2}}\right\}\right]
\end{aligned}
$$

where 


$$
u_{i}=\int_{0}^{1} \alpha_{i}(t) d x(t), \quad \xi_{i}=\int_{0}^{1} \alpha_{i}(t) d x_{0}(t), \quad i=1,2, \cdots
$$

We now substitute (2.6) and (2.3) and obtain

$$
\begin{aligned}
& \sum_{m_{1}, \cdots, m_{N}=0}^{\infty} \lambda^{m_{1}+\cdots+m_{N}} A_{m_{1}}, \cdots, m_{N} \Psi_{m_{1}}, \cdots, m_{N}\left(x_{0}\right) \\
= & \left(1-\lambda^{2}\right)^{-N / 2} \int_{C}^{w} F(x) \exp \left[\sum_{i=1}^{N} \frac{2 \lambda u_{i} \xi_{i}-\lambda^{2}\left(\underline{u}_{i}^{2}+\xi_{i}^{2}\right)}{1-\lambda^{2}}\right] d_{w} x .
\end{aligned}
$$

If we specialize $F(x)$ for the moment by making $F(x) \equiv 1$, then since $\Psi_{0,0, \cdots, 0}=\Psi_{0}=H_{0} \equiv 1$, and all the other $\Psi^{\prime}$ 's are orthogonal to $\Psi_{0}$, we have $A_{0,0, \cdots, 0}=1$, and all the other $A$ 's are zero. Hence

$$
\left(1-\lambda^{2}\right)^{-N / 2} \int_{C}^{w} 1 \cdot \exp \left[\sum_{i=1}^{N} \frac{2 \lambda u_{i} \xi_{i}-\lambda^{2}\left(u_{i}^{2}+\xi_{i}^{2}\right)}{1-\lambda^{2}}\right] d_{w} x=1
$$

with $u_{i}$ given by $(2.7)$. Then we may multiply through by $F\left(x_{0}\right)$ in (2.9), subtract from (2.8), and obtain

$$
\begin{aligned}
& D\left(x_{0}\right) \equiv \sum_{m_{1}, \cdots, m_{N}=0}^{\infty} \lambda^{{ }^{m_{1}+\cdots+m_{N}} A_{m_{1}}, \cdots, m_{N} \Psi_{m_{1}}, \cdots, m_{N}}\left(x_{0}\right)-F\left(x_{0}\right) \\
&=c_{\lambda} \int_{C}^{w}\left[F(x)-F\left(x_{0}\right)\right] \exp \left[\sum_{i=1}^{N} \frac{2 \lambda u_{i} \xi_{i}-\lambda^{2}\left(u_{i}^{2}+\xi_{i}^{2}\right)}{1-\lambda^{2}}\right] d_{w} x,
\end{aligned}
$$

where

$$
c_{\lambda}=\left(1-\lambda^{2}\right)^{-N / 2} \text {. }
$$

We have now obtained an integral expression for the difference $D\left(x_{0}\right)$ between a functional and its Fourier-Hermite partial sum, and we shall establish in the following sections that $D\left(x_{0}\right) \rightarrow 0$ as $\lambda \rightarrow 1-0$ and $N \rightarrow \infty$.

3. Domination of $D\left(x_{0}\right)$ by a sum of integrals $I$. To prove that $D\left(x_{0}\right) \rightarrow 0$ as $\lambda \rightarrow 1-0$ and $N \rightarrow \infty$, we first choose an arbitrary $\epsilon>0$, then break up our space $C$ and express it as a sum of sets $S_{j}$ characterized by certain inequalities, and finally dominate $D\left(x_{0}\right)$ by a sum of integrals $I_{j}$ taken over the sets $S_{j}$.

Choose $\epsilon>0$. Then there exists a $\delta>0$ such that if

$$
\int_{0}^{1}\left[x(t)-x_{0}(t)\right]^{2} d t \leqq \delta^{2}, \quad\left|F(x)-F\left(x_{0}\right)\right|<\epsilon / 3 .
$$


We let $S_{0}$ denote the set of functions $x(t)$ satisfying this first inequality:

$$
S_{0}=\underset{x(t)}{\mathrm{E}}\left\{\int_{0}^{1}\left[x(t)-x_{0}(t)\right]^{2} d t \leqq \delta^{2}\right\} .
$$

The complement $S_{0}^{-1}$ of $S_{0}$ will then consist of all $x(t)$ satisfying the reverse inequality:

$$
S_{0}^{-1}=\underset{x(t)}{\mathrm{E}}\left\{\int_{0}^{1}\left[x(t)-x_{0}(t)\right]^{2} d t>\delta^{2}\right\} .
$$

Let

$$
\mu=\frac{\delta}{2}\left(1+\int_{0}^{1} x_{0}^{2}(t) d t\right)^{-1 / 2}
$$

so that

$$
\mu^{2} \int_{0}^{1} x_{0}^{2}(t) d t<\frac{\delta^{2}}{4}
$$

Choose $N_{0}$ so great that

$$
\sum_{j=N_{0}+1}^{\infty}\left(\int_{0}^{1} \beta_{j}(t) x_{0}(t) d t\right)^{2}<\frac{\delta^{2}}{16}
$$

where

$$
\beta_{j}(t)=2^{1 / 2} \sin (j-1 / 2) \pi t, \quad j=1,2, \cdots .
$$

This can be done since this sum is just the latter part of the (necessarily convergent) Parseval sum for $\int_{0}^{1} x_{0}^{2}(t) d t$. Now let $x_{1}(t)$ be the finite sum

$$
x_{1}(t)=\sum_{j=1}^{N 0} \beta_{j}(t) \int_{0}^{1} \beta_{j}(s) x_{0}(s) d s,
$$

and $x_{2}(t)=x_{0}(t)-x_{1}(t)$. Thus

$$
\begin{aligned}
& \int_{0}^{1} \beta_{j}(t) x_{2}(t) d t=\left\{\begin{array}{l}
0 \text { if } j \leqq N_{0} \\
\int_{0}^{1} \beta_{j}(t) x_{0}(t) d t \text { if } j>N_{0},
\end{array}\right. \\
& \int_{0}^{1} x_{2}^{2}(t) d t=\sum_{j=N+1}^{\infty}\left[\int_{0}^{1} \beta_{j}(t) x_{0}(t) d t\right]^{2}<\frac{\delta^{2}}{16} .
\end{aligned}
$$


We now have from (3.2), (3.3), and (3.7) that when $x(t) \in S_{0}^{-1}$,

$$
\int_{0}^{1}\left[x(t)-x_{0}(t)\right]^{2} d t-\mu^{2} \int_{0}^{1} x_{0}^{2}(t) d t-4 \int_{0}^{1} x_{2}^{2}(t) d t>\frac{\delta^{2}}{2}
$$

or by the Parseval equation

$$
\begin{aligned}
\sum_{j=1}^{\infty}\left[\left\{\int_{0}^{1} \beta_{j}(t)\left[x(t)-x_{0}(t)\right] d t\right\}^{2}-\mu^{2}\right. & \left\{\int_{0}^{1} \beta_{j}(t) x_{0}(t) d t\right\}^{2} \\
& \left.-4\left\{\int_{0}^{1} \beta_{j}(t) x_{2}(t) d t\right\}^{2}\right]>\frac{\delta^{2}}{2}
\end{aligned}
$$

and by integration by parts (using (1.4) and (3.5)),

$$
\sum_{j=1}^{\infty} \frac{\left\{\int_{0}^{1} \alpha_{j}(t) d\left[x(t)-x_{0}(t)\right]\right\}^{2}-\mu^{2}\left\{\int_{0}^{1} \alpha_{j}(t) d x_{0}(t)\right\}^{2}-4\left\{\int_{0}^{1} \alpha_{j}(t) d x_{2}(t)\right\}^{2}}{\pi^{2}(j-1 / 2)^{2}}>\frac{\delta^{2}}{2} .
$$

Note that the sum on the left would still converge if the numerator were independent of $j$ and the denominator involved $(j-1 / 2)$ to the $3 / 2$ power only. Let us therefore investigate the least upper bound of the product of the numerator and $(j-1 / 2)^{-1 / 2}$ : that is, let

$$
\eta=\sup _{j}\left[\frac{\left\{\int_{0}^{1} \alpha_{j}(t) d\left[x(t)-x_{0}(t)\right]\right\}^{2}-\mu^{2}\left\{\int_{0}^{1} \alpha_{j}(t) d x_{0}(t)\right\}-4\left\{\int_{0}^{1} \alpha_{j}(t) d x_{2}(t)\right\}^{2}}{(j-1 / 2)^{1 / 2}}\right] .
$$

Then we have (whether $\eta$ is finite or $+\infty$ )

$$
\sum_{j=1}^{\infty} \frac{\eta}{\pi^{2}(j-1 / 2)^{3 / 2}}>\frac{\delta^{2}}{2}
$$

and

$$
\eta>\frac{\pi^{2} \delta^{2}}{2 \sum_{j=1}^{\infty}\left(j-\frac{1}{2}\right)^{-3 / 2}} .
$$

But

$$
\begin{aligned}
\sum_{j=1}^{\infty}\left(j-\frac{1}{2}\right)^{-3 / 2} & =\left(\frac{1}{2}\right)^{-3 / 2}+\sum_{j=2}^{\infty}\left(j-\frac{1}{2}\right)^{-3 / 2} \\
& <2^{3 / 2}+\int_{1}^{\infty}\left(s-\frac{1}{2}\right)^{-3 / 2} d s \\
& =2^{3 / 2}+2^{3 / 2}<6
\end{aligned}
$$

so 


$$
\eta>\pi^{2} \delta^{2} / 12>\delta^{2} / 2 .
$$

By definition of $\eta$, whether $\eta$ is finite or infinite, there must exist some value of $i$, say $i=i_{0}$, for which

$$
\frac{\delta^{2}}{2}<\frac{\left\{\int_{0}^{1} \alpha_{i}(t) d\left[x(t)-x_{0}(t)\right]\right\}^{2}-\mu^{2}\left\{\int_{0}^{1} \alpha_{i}(t) d x_{0}(t)\right\}^{2}-4\left\{\int_{0}^{1} \alpha_{i}(t) d x_{2}(t)\right\}^{2}}{(i-1 / 2)^{1 / 2}},
$$

so that if

(3.8) $u_{i}=\int_{0}^{1} \alpha_{i}(t) d x(t), \quad \xi_{i}=\int_{0}^{1} \alpha_{i}(t) d x_{0}(t), \quad \xi_{i}^{\prime}=\int_{0}^{1} \alpha_{i}(t) d x_{2}(t)$,

we have for $x \in S_{0}^{-1}$ and some corresponding value of $i$ (namely, the $i_{0}$ chosen above),

$$
\left(u_{i}-\xi_{i}\right)^{2}>\frac{\delta^{2}}{2}\left(i-\frac{1}{2}\right)^{1 / 2}+\mu^{2} \xi_{i}^{2}+4 \xi_{i}^{\prime 2}
$$

Since

$$
(a+b+c)^{2} \leqq 3\left(a^{2}+b^{2}+c^{2}\right),
$$

we have

$$
\begin{aligned}
\left|u_{i}-\xi_{i}\right| & >\frac{1}{3^{1 / 2}}\left[\frac{\delta}{2^{1 / 2}}\left(i-\frac{1}{2}\right)^{1 / 4}+\mu\left|\xi_{i}\right|+2\left|\xi_{i}{ }^{\prime}\right|\right] \\
& >\frac{\delta}{3}\left(i-\frac{1}{2}\right)^{1 / 4}+\frac{1}{2} \mu\left|\xi_{i}\right|+\left|\xi_{i}{ }^{\prime}\right| .
\end{aligned}
$$

Thus if we denote by $S_{j}$ the set

$$
\begin{aligned}
S_{j} & =\underset{x(t)}{\mathrm{E}}\left\{\left|\int_{0}^{1} \alpha_{j}(t) d x(t)-\xi_{j}\right|\right. \\
> & \left.\frac{\delta}{3}\left(j-\frac{1}{2}\right)^{1 / 4}+\frac{1}{2} \mu\left|\xi_{j}\right|+\left|\xi_{j}^{\prime}\right|\right\}
\end{aligned}
$$

for $j=1,2,3, \cdots$, we have

$$
S_{0}^{-1} \subset \sum_{j=1}^{\infty} S_{j}
$$

and

$$
C=S_{0}+\sum_{j=1}^{\infty} S_{j}=\sum_{j=0}^{\infty} S_{j}
$$


Now if we let

$$
\begin{aligned}
& I_{j}=c_{\lambda} \int_{S_{j}}^{w}\left|F(x)-F\left(x_{0}\right)\right| \\
& \cdot \exp \left\{\sum_{i=1}^{N} \frac{2 \lambda u_{i} \xi_{i}-\lambda^{2}\left(u_{i}^{2}+\xi_{i}^{2}\right)}{1-\lambda^{2}}\right\} d_{w} x,
\end{aligned}
$$

then we have by (2.10) and (3.10),

$$
\left|D\left(x_{0}\right)\right| \leqq \sum_{j=0}^{\infty} I_{j}
$$

In the following sections we shall obtain suitable estimates for the integrals $I_{j}$.

4. One-dimensional integral expressions for the Wiener integrals $I_{j}$. It is easy to see from (3.1), (2.9), and (2.11) that for $I_{0}$ we shall have

$$
\begin{aligned}
I_{0} & <\frac{\epsilon}{3} c_{\lambda} \int_{S_{0}}^{w} \exp \left\{\sum_{i=1}^{N} \frac{2 u_{i} \xi_{i} \lambda-\lambda^{2}\left(u_{i}^{2}+\xi_{i}^{2}\right)}{1-\lambda^{2}}\right\} d_{w} x \\
& \leqq \frac{\epsilon}{3} c_{\lambda} \int_{C}^{w} \exp \left\{\sum_{i=1}^{N} \frac{2 u_{i} \xi_{i} \lambda-\lambda^{2}\left(u_{i}^{2}+\xi_{i}^{2}\right)}{1-\lambda^{2}}\right\} d_{w} x=\frac{\epsilon}{3} .
\end{aligned}
$$

Consider now $I_{j}$, for $j>0$. Since $F(x)$ is bounded by hypothesis, there exists a number $B$ such that $|F(x)| \leqq B$. Then from (3.11)

$$
\begin{aligned}
I_{j} & \leqq 2 c_{\lambda} B \int_{S_{j}}^{w} \exp \left\{\sum_{i=1}^{N} \frac{2 \lambda u_{i} \xi_{i}-\lambda^{2}\left(u_{i}^{2}+\xi_{i}^{2}\right)}{1-\lambda^{2}}\right\} d_{w} x \\
& =2 c_{\lambda} B \int_{S_{i}}^{w} \exp \left\{\sum_{i=1}^{N} u_{i}^{2}\right\} \exp \left\{-\sum_{i=1}^{N} \frac{\left(\lambda \xi_{i}-u_{i}\right)^{2}}{1-\lambda^{2}}\right\} d_{w} x .
\end{aligned}
$$

The Wiener integral on the right depends on the $u_{i}$ as $i=1,2, \cdots, N$ and on $u_{j}$; that is, it depends on $N$ linear Stieltjes integrals

$$
u_{k}=\int_{0}^{1} \alpha_{k}(t) d x(t), \quad k=1,2, \cdots, N,
$$

if $j \leqq N$, while if $j>N$, it depends also on the additional

$$
u_{j}=\int_{0}^{1} \alpha_{j}(t) d x(t) .
$$


These Wiener integrals can be evaluated by (1.1), and present correspondingly an $N$ - or $(N+1)$-fold Lebesgue integral. Let now

(4.2) $\chi_{j}(v)=\left\{\begin{array}{l}1, \text { if }\left|v-\xi_{j}\right|>\frac{\delta}{3}\left(j-\frac{1}{2}\right)^{1 / 4}+\frac{1}{2} \mu\left|\xi_{j}\right|+\left|\xi_{j}^{\prime}\right|, \\ 0, \text { otherwise. }\end{array}\right.$

Then we have by (3.9), (3.8), and (4.2)

$$
I_{j} \leqq 2 B c_{\lambda} \pi^{-(N+1) / 2} \int_{-\infty}^{\infty} \cdots \int_{-\infty}^{\infty} \chi_{j}\left(v_{j}\right) e^{-v_{i}^{2}}
$$

$$
\begin{aligned}
& \cdot \exp \left\{-\sum_{i=1}^{N} \frac{\left(\lambda \xi_{i}-v_{i}\right)^{2}}{1-\lambda^{2}}\right\} d v_{1} \cdots d v_{N} d v_{j}(j>N), \\
& I_{i} \leqq 2 B c_{\lambda} \pi^{-N / 2} \int_{-\infty}^{\infty} \cdots \int_{-\infty}^{\infty} \chi_{j}\left(v_{j}\right) \\
& \cdot \exp \left\{-\sum_{i=1}^{N} \frac{\left(\lambda \xi_{i}-v_{i}\right)^{2}}{1-\lambda^{2}}\right\} d v_{1} \cdots d v_{N} \quad(j \leqq N) .
\end{aligned}
$$

In the case of the $(N+1)$-fold integral of (4.3), we have $N$ factors of the form

$$
\int_{-\infty}^{\infty} \exp \left\{-\frac{\left(\lambda \xi_{i}-v\right)^{2}}{1-\lambda^{2}}\right\} d v
$$

and one of the form

$$
\int_{-\infty}^{\infty} \chi_{j}(v) e^{-v^{2}} d v
$$

For the $N$-fold integral of (4.4), there are $N-1$ factors of the type (4.5) and the other one (for $v_{j}$ ) is

$$
\int_{-\infty}^{\infty} \chi_{j}(v) \exp \left\{-\frac{\left(\lambda \xi_{j}-v\right)^{2}}{1-\lambda^{2}}\right\} d v
$$

The integrals of type (4.5) are easily evaluated, and we get for each one $\left(\pi\left(1-\lambda^{2}\right)\right)^{1 / 2}$. Thus we have (remembering (2.11)),
(4.6) $\quad I_{j} \leqq \frac{2 B}{\pi^{1 / 2}} \int_{-\infty}^{\infty} \chi_{j}(v) e^{-v^{2}} d v$
$(j>N)$,

$$
I_{j} \leqq \frac{2 B}{\left(\pi\left(1-\lambda^{2}\right)\right)^{1 / 2}} \int_{-\infty}^{\infty} \chi_{j}(v) \exp \left\{-\frac{\left(\lambda \xi_{j}-v\right)^{2}}{1-\lambda^{2}}\right\} d v \quad(j \leqq N)
$$


5. Estimation of $I_{j}$ for the case $j>N$. In this section we shall refine our estimate for $I_{j}$ for $j>N$. We take now $N>N_{0}$ (see (3.4)) and note that since $j>N>N_{0}$, we have, by (3.6) and (3.8), $\xi_{j}^{\prime}=\xi_{j}$, and hence by (4.2) if $\chi_{j}(v)=1$,

$$
\begin{aligned}
\left|v-\xi_{j}\right| & >\frac{\delta}{3}\left(j-\frac{1}{2}\right)^{1 / 4}+\frac{1}{2} \mu\left|\xi_{j}\right|+\left|\xi_{j}\right| \\
& \geqq \frac{\delta}{3}\left(j-\frac{1}{2}\right)^{1 / 4}+\left|\xi_{j}\right| .
\end{aligned}
$$

Thus, $\chi_{j}(v)=1$ implies that

$$
|v| \geqq \frac{\delta}{3}\left(j-\frac{1}{2}\right)^{1 / 4},
$$

so that we don't decrease the integral in (4.6) when we drop $\chi_{3}(v)$ and integrate over the $v$ defined by (5.1). Thus

$$
I_{j} \leqq \frac{2 B}{\pi^{1 / 2}} \cdot 2 \int_{\delta(j-1 / 2)^{1 / 4} / 3}^{\infty} e^{-v^{2}} d v=\frac{4 B}{\pi^{1 / 2}} \operatorname{erfc}\left\{\frac{\delta}{3}\left(j-\frac{1}{2}\right)^{1 / 4}\right\}
$$

where

$$
\operatorname{erfc}(M)=\int_{M}^{\infty} e^{-t^{2}} d t
$$

We note that this estimate of $I_{j}$ is independent of $\lambda$.

6. Estimation of $I_{j}$ for the case $j \leqq N$. In order to reffne our estimate of $I_{j}$ in this case we make a change of variable in (4.7):

$$
s=\frac{v-\lambda \xi_{j}}{\left(1-\lambda^{2}\right)^{1 / 2}}
$$

so that the integral of (4.7) becomes

$$
\begin{aligned}
\int_{-\infty}^{\infty} \chi_{j}(v) \exp \{- & \left.-\frac{\left(v-\lambda \xi_{j}\right)^{2}}{1-\lambda^{2}}\right\} d v \\
& =\left(1-\lambda^{2}\right)^{1 / 2} \int_{-\infty}^{\infty} \chi_{j}\left(s\left(1-\lambda^{2}\right)^{1 / 2}+\lambda \xi_{j}\right) e^{-s^{2}} d s
\end{aligned}
$$

If $1>\lambda>1-\mu / 2$, then

$$
(1-\lambda)\left|\xi_{j}\right| \leqq 2^{-1} \mu\left|\xi_{j}\right|,
$$

and if $\chi_{j}\left(s\left(1-\lambda^{2}\right)^{1 / 2}+\lambda \xi_{j}\right)=1$, we have from (4.2) 


$$
\begin{aligned}
\left|s\left(1-\lambda^{2}\right)^{1 / 2}+(\lambda-1) \xi_{j}\right| & >\frac{\delta}{3}\left(j-\frac{1}{2}\right)^{1 / 4}+\frac{\mu}{2}\left|\xi_{j}\right|+\left|\xi_{j}^{\prime}\right| \\
& \geqq \frac{\delta}{3}\left(j-\frac{1}{2}\right)^{1 / 4}+\frac{\mu}{2}\left|\xi_{j}\right| .
\end{aligned}
$$

Thus

$$
\left|s\left(1-\lambda^{2}\right)^{1 / 2}\right| \geqq \frac{\delta}{3}\left(j-\frac{1}{2}\right)^{1 / 4}
$$

that is,

$$
|s| \geqq \frac{\delta(j-1 / 2)^{1 / 4}}{3\left(1-\lambda^{2}\right)^{1 / 2}}
$$

so that we don't decrease the integral in (6.1) when we drop $\chi_{j}$ and integrate over the set of $s$ defined by (6.2). Hence we obtain for $j \leqq N$ and $1>\lambda>1-\mu / 2$

$$
I_{j} \leqq \frac{4 B}{\pi^{1 / 2}} \int_{\delta(j-1 / 2)^{1 / 4 / 3\left(1-\lambda^{2}\right)^{1 / 2}}}^{\infty} e^{-\delta^{2}} d s=\frac{4 B}{\pi^{1 / 2}} \operatorname{erfc}\left\{\frac{\delta(j-1 / 2)^{1 / 4}}{3\left(1-\lambda^{2}\right)^{1 / 2}}\right\}
$$

7. The final estimate for $D\left(x_{0}\right)$. We now combine our estimates for $I_{j}$, making both assumptions of $\S \S 5$ and 6 ; in particular, that $N>N_{0}$ and $1>\lambda>1-\mu / 2$. Then (5.2) holds for $j>N$ and (6.3) for $j \leqq N$. We have then

$$
\begin{aligned}
\sum_{j=1}^{\infty} I_{j} \leqq & \frac{4 B}{\pi^{1 / 2}}\left[\sum_{j=1}^{N} \operatorname{erfc}\left\{\frac{\delta(j-1 / 2)^{1 / 4}}{3\left(1-\lambda^{2}\right)^{1 / 2}}\right\}\right. \\
& \left.+\sum_{j=N+1}^{\infty} \operatorname{erfc}\left\{\frac{\delta}{3}\left(j-\frac{1}{2}\right)^{1 / 4}\right\}\right] .
\end{aligned}
$$

Since

$$
\operatorname{erfc}(M)=\int_{M}^{\infty} e^{-t^{2}} d t<\frac{e^{-M^{2}}}{2 M}
$$

it follows that

$$
\begin{aligned}
\sum_{j=1}^{\infty} I_{j}< & \frac{2 B}{\pi^{1 / 2}} \sum_{j=1}^{\infty} \frac{3\left(1-\lambda^{2}\right)^{1 / 2}}{\delta(j-1 / 2)^{1 / 4}} \exp \left\{\frac{-\delta^{2}(j-1 / 2)^{1 / 2}}{9\left(1-\lambda^{2}\right)}\right\} \\
& +\frac{2 B}{\pi^{1 / 2}} \sum_{j=N+1}^{\infty} \frac{3}{\delta(j-1 / 2)^{1 / 4}} \exp \left\{\frac{-\delta^{2}(j-1 / 2)^{1 / 2}}{9}\right\}
\end{aligned}
$$


But the sum in the second term on the right above converges, so that we may take $N_{\epsilon}\left(\geqq N_{0}\right)$ large enough to make this term less than $\epsilon / 3$ for $N>N_{\epsilon}$. Moreover, the series of the first term on the right converges for all $\lambda, 0<\lambda<1$, and each term of this sum is decreasing in $\lambda$ as $\lambda \rightarrow 1-0$, and has the limit zero. Thus the first term on the right of (7.1) has the limit zero. We may therefore choose $\lambda_{\epsilon}$ in the interval $(1-\mu / 2,1)$ so that the first term is less than $\epsilon / 3$ when $\lambda_{\epsilon}<\lambda<1$. Thus when $N>N_{\epsilon}$ and $\lambda_{\epsilon}<\lambda<1$ we have from (3.12) and (4.1),

$$
\left|D\left(x_{0}\right)\right| \leqq \sum_{j=0}^{\infty} I_{j}<\epsilon .
$$

Referring to the theorem of $\S 1$ and the definition of $D\left(x_{0}\right)$ in (2.10), we see that the theorem is established.

8. Proof of the corollary. The regularity of the Abel mean for the one-dimensional case, together with the absolute convergence of (1.5) assumed in the hypothesis of the corollary, at once permit us to write

$$
\begin{aligned}
\lim _{\lambda \rightarrow 1 \rightarrow 0} \sum_{n=0}^{\infty} \lambda^{n} \sum_{m_{1}+\cdots+m_{N}=n} A_{m_{1}}, \cdots, m_{N} & \Psi_{m_{1}}, \cdots, m_{N}\left(x_{0}\right) \\
& =\sum_{n=0}^{\infty} \sum_{m_{1}+\cdots+m_{N}=n} A_{m_{1}, \cdots, m_{N}} \Psi_{m_{1}, \cdots, m_{N}}\left(x_{0}\right) \\
& =\sum_{m_{1}, \cdots, m_{N}=0}^{\infty} A_{m_{1}, \cdots, m_{N}} \Psi_{m_{1}, \cdots, m_{N}}\left(x_{0}\right) .
\end{aligned}
$$

Now let $\epsilon>0$. Then by the theorem there exists an $N_{0}>0$ and a $\lambda_{0}$ in $(0,1)$ such that when $N>N_{0}$ and $\lambda_{0}<\lambda<1$,

$$
\left|F\left(x_{0}\right)-\sum_{m_{1}, \cdots, m_{N}=0}^{\infty} A_{m_{1}, \cdots, m_{N}} \lambda^{m_{1}+\cdots+m_{N} \Psi_{m_{1}}, \cdots, m_{N}}\left(x_{0}\right)\right|<\epsilon .
$$

Taking the limit with respect to $\lambda$ in (8.1) we get

$$
\left|F\left(x_{0}\right)-\sum_{m_{1}, \cdots, m_{N}=0}^{\infty} A_{m_{1}, \cdots, m_{N}} \Psi_{m_{1}, \cdots, m_{N}}\left(x_{0}\right)\right| \leqq \epsilon .
$$

But this establishes (1.6), and hence the corollary.

The University of Minnesota 